\title{
Reference values of 6 minutes walk test (6 MWT) in Bangladeshi healthy subjects aged 25-55 years
}

\author{
Mosharraf-Hossain AKM, Chakrabortty R \\ Department of Respiratory Medicine, Bangabandhu Sheikh Mujib Medical University, \\ Shahbag, Dhaka. Email: akmmosharrafhossain@yahoo.com
}

\begin{abstract}
Introduction: The six minute walk test (6MWT) is increasingly accepted worldwide to assess functional exercise capacity of cardiac and pulmonary diseases for its simplicity. Aims and objectives: The aim of the present study was to provide reference values for 6 Minute Walk Distance (6MWD) and other primary variables such as oxygen saturation $\left(\mathrm{SaO}_{2}\right)$, pulse rate $(\mathrm{PR})$ and breathlessness perception done in 6MWT. Methods: We studied a sample of 190 healthy subjects (53 females) of age between 25-55 years (37.9 \pm 8.5 years). Baseline lung functions including forced expiratory volume in $1^{\text {st }}$ second (FEV1), forced vital capacity (FVC) and FEV1/FVC ratio were measured by a flow sensing spirometer. All subjects performed 6MWT according to standard protocol provided by the American Thoracic Society (ATS) guidelines. The fatigue and dyspnoea was measured before and after 6MWT by modified Borg scale. The $\mathrm{SaO}_{2}$ and PR were measured also by a light weight pulse oxymeter. Results: The mean 6MWD was $466.7 \pm 69.4$ meter, ranging from 213 to 659 meter and the mean Distance Work (DW) was $28732 \pm 7024 \mathrm{~kg}-\mathrm{m}$. Dyspnoea status and oxygen saturation remained unaltered throughout the walk. Mean resting and walking $\mathrm{SaO}_{2}$ values were, respectively, 97.1 $\pm 1.3 \%$ and $96.8 \pm 1.5 \%$. The mean change in $\mathrm{SaO}_{2}$ was $3 \pm 1.5 \%$. PR was significantly affected by the walk. Mean resting and maximum PR after walking were respectively, $80 \pm 7$ and $103 \pm 12 \mathrm{bpm}(\mathrm{p}<.01)$. Gender significantly affected the 6MWD ( $\mathrm{p}<.01)$. In all subjects, the 6MWD was inversely and directly related, respectively, to age $(\mathrm{r}=-.15 ; \mathrm{P}<.05)$ and height $(\mathrm{r}=.41 ; \mathrm{P}<.01)$. Conclusion: This study showed reference values for the 6MWT variables of subjects in Bangladeshi population.
\end{abstract}

\section{Introduction}

Exercise testing is increasingly utilized in clinical practice to optimize patient management and acquire valuable functional and prognostic information not obtainable through static cardiopulmonary measurements. A range of exercise testing protocols are available for this purpose; among them walking distance is an economical and expeditious gauge of functional ability. ${ }^{1}$ It provides a comprehensive assessment of all the mechanisms involved during exercise including the cardiovascular, respiratory, neurolocomotor and the metabolic systems. Walking is the natural advancement of a subject through space with minimal mechanical and physiological expenditure. Six-minute walk test (6MWT) in this respect is a simple, low tech, easy to perform reproducible test. ${ }^{1,2}$ It entails measuring the maximal distance that a subject can travel during a self-paced walk on a flat hard surface over a 6 minutes period. It has shown to reflect the capacity to undertake day-to-day activities and correlates well with health related quality of life scores. ${ }^{3}$ Additionally, among patients with moderate to severe $\mathrm{COPD},{ }^{4}$ and congestive heart failure, ${ }^{5}$ the total distances covered during the 6MWT has shown a moderately strong correlation with the peak oxygen uptake measured during an incremental cycle ergometry test. A reduction in walking distance is frequently multifactorial and may result from a varied set of disorders that afflict a series of physiological variables engaged during exercise. ${ }^{6}$ It is the enormity of these factors that make the assessment of walking capacity useful in such a wide ranging and diverse set of situations. However, the most valuable clinically important indications remain; measuring and comparing outcomes pre and post-treatment, evaluation of functional status and predicting morbidity and mortality. ${ }^{2}$ A potential limitation in the application of 6MWT is the dearth of reference values for a variety of race/ethnic groups in both genders and across a wide range of ages, ${ }^{7}$ which could prove useful in evaluating disability, prognosis, conducting research and judging therapeutic interventions. Moreover, there is evidence 
supporting a variation in 6MWD among different population groups and within ethnic/racial groups. For example, healthy African Americans have shown to walk on average $40 \mathrm{~m}$ less than white Americans after correcting for age, gender, height and weight. ${ }^{8}$ It is, therefore, plausible that such diversity also exist among different South East Asian populations. As far as we are aware, there are few published data on the normal 6MWD for South East Asian community. The purpose of this study was to measure the distance covered by a healthy Bangladesh adult in six minutes and provide insight into physiological determinants of the 6MWD.

\section{Materials and Methods}

This study was conducted at Respiratory Medicine Wing of Bangabadhu Sheikh Mujib Medical University (BSMMU), Shahbagh, Dhaka, Bangladesh from July 2012 to June 2013. The study was conducted in compliance with the 'Ethical Principles for Medical Research involving Human Subjects' of Helsinki Declaration. ${ }^{9} \quad$ Written and informed consent was obtained prior to participation. This prospective study randomly enrolled volunteering healthy Bangladeshi individuals aged between $25-55$ years. The subjects included staff members at the BSMMU hospitals, attendants of patients and personnel undergoing routine employment and health screening at hospital's various inpatient and outpatient Departments. The exclusion criteria were i) $>10$ pack years of smoking; ii) BMI >30 iii) Chronic pulmonary diseases like bronchial asthma, COPD etc and any 6MWT contraindications like recent MI, osteoarthritis etc. One Research Assistant was appointed and properly trained to perform the procedure. A standardized protocol based on published guidelines was used in the conduct of the 6 MWT. $^{2}$

The comparison of factors between gender will be done using Student $\mathrm{t}$ test. Potential factors including age, height, weight, body mass index [BMI], FEV1, \% FEV1 predicted, FVC, \% FVC predicted, FEV1/FVC ratio, resting heart rate, end of exercise heart rate, difference in heart rate before and after exercise, resting $\mathrm{SaO}_{2}$, end of exercise $\mathrm{SaO}_{2}$, difference in $\mathrm{SaO}_{2}$ before and after exercise will be evaluated for their association with 6WMD by first univariate analysis with the Spearman's correlation test and then adjusted to multivariate analysis using the forward stepwise multiple linear regression. Analysis was performed using Statistical Package of Social Science (SPSS version 16.0). The level of significance will be set at $5 \%$ in all comparisons.

\section{Results}

Table I shows the mean 6MWD was $466.7 \pm 69.4 \mathrm{~m}$, ranging from 213 to $659 \mathrm{~m}$ and the mean DW was $28732 \pm 7024 \mathrm{~kg}-\mathrm{m}$. Dyspnoea status and oxygen saturation remained unaltered throughout the walk. Mean resting and walking $\mathrm{SaO}_{2}$ values were, respectively, $97.1 \pm 1.3 \%$ and $96.8 \pm 1.5 \%$. The mean change in $\mathrm{SaO}_{2}$ was $3 \pm 1.5 \%$. PR was significantly affected by the walk. Mean resting and maximum PR after walking were respectively, $80 \pm 7$ and $103 \pm 12 \mathrm{bpm}(\mathrm{p}<.01)$. Gender significantly affected the 6MWD $(\mathrm{p}<.01)$. In all subjects, the 6MWD was inversely and directly related, respectively, to age $(\mathrm{r}=-.15 ; \mathrm{P}<.05)$ and height $(\mathrm{r}=.41 ; \mathrm{P}<.01)$.

Table 1: Characteristics and 6 MWT measures of study subjects

\begin{tabular}{lllc}
\hline Characteristics & Male $(\mathrm{n}=147)$ & Female $(\mathrm{n}=53)$ & P value \\
\hline Age, years & $37.2 \pm 7.2$ & $39.7 \pm 11.1$ & $<.05$ \\
Height, cm & $169.6 \pm 7.8$ & $151 \pm 4.8$ & $<.01$ \\
Weight, $\mathrm{kg}$ & $64.3 \pm 8.4$ & $53.2 \pm 9.6$ & $<.01$ \\
6MWD, m & $487.5 \pm 51.8$ & $413.1 \pm 80.1$ & $<.01$ \\
DW, kg-m & $31266 \pm 5357$ & $22181 \pm 6602$ & $<.01$ \\
PR baseline & $80 \pm 7$ & $82 \pm 9$ & $<.05$ \\
PR maximal & $105 \pm 12$ & $100 \pm 11$ & $<.05$ \\
\hline
\end{tabular}

\section{Discussion}

To the authors' knowledge, data from healthy Bangladeshi adult in literature on reference values and factors influencing 6MWT and 6MWD are inadequate. In the present study the mean 6MWD was $487.5 \pm 51.8$ and $413.1 \pm 80.1 \mathrm{~m}$ for men and women respectably. Age and gender were the significant independent predictors of 6MWD and together accounted for $15 \%$ of the variance in the distance walked.

There is considerable variation between published references for 6MWD. Among Asian population, the mean 6MWD for healthy Singaporean men and women (mean age 61 years) was $580 \mathrm{~m}$ and $538 \mathrm{~m}$ respectively. ${ }^{10}$ Similarly, for a healthy cohort of Japanese men and women (mean age 65 years) reported a mean 6MWD of $572 \mathrm{~m}$ and $504 \mathrm{~m}^{11}$ and a mean value of $624 \mathrm{~m}$ and $541 \mathrm{~m}$ respectively. ${ }^{12}$ For Caucasian based studies, Troosters (mean age 65 years), ${ }^{13}$ and Enright (mean age 67 years) ${ }^{8}$ published a mean 6MWD for a healthy cohort of $673 \mathrm{~m}$ and $400 \mathrm{~m}$ for men and $589 \mathrm{~m}$ and $367 \mathrm{~m}$ for women respectively. A study from Tucson, USA on 290 healthy adults (mean age 60 years) demonstrated a 6MWD of $466 \mathrm{~m}$ for men and 544 $\mathrm{m}$ for women. ${ }^{14}$ Similarly, Rikli in 7183 senior men and women reported values of $689 \mathrm{~m}$ and $624 \mathrm{~m}$ respectively. ${ }^{15}$ Several factors that have been put forward in explaining this wide variation in the 
published data include difference in race and ethnicity, subject selection and testing methodologies.

There are several potential explanations for these discrepancies. First, in terms of testing strategy, the length of the walking course i.e. $45 \mathrm{ft} / 13.3 \mathrm{~m}$, meant that subjects had to turn more frequently and thereby also consume more time in reversing directions, leading to a shorter 6MWD. Although, American Thoracic Society (ATS) recommends 30 $\mathrm{m}$ walking course, ${ }^{2}$ investigators have employed corridors ranging from $15 \mathrm{~m}$ to $50 \mathrm{~m}$ in published studies. $^{13,16}$ Second, a learning effect of repeat testing or a practice session has been demonstrated to significantly impact the 6MWD in previous studies. $^{13,17}$ An ATS review of published data reported this to be from a mean of zero to $17 \%$. $^{6}$ Since these subjects were naive to the walk test and were not allowed a practice session, this impacted the distance covered in six minutes by our cohort. A third factor would be subject-related differences in terms of racial characteristics, motivation and psychological factors, exercise habits, coordination and nutritional status. Analogous to previously published data, ${ }^{10,13,14,17}$ the physiological determinants of height and weight showed a direct, where as age, an inverse correlation with 6MWD in our subjects. A plausible explanation for a taller person would be the taking of longer length strides resulting in less time spent in contact with the ground plus shorter periods supported by two legs, thereby improving gait efficiency. Similarly, a heavier individual would require additional energy while ambulating to support an enlarged body mass (increase in adipose tissue rather than muscle mass) and thereby curtailing the maximal level of effective work. ${ }^{18}$ Moreover, an inverse relation between advancing age and 6MWD most likely represents a combination of loss in the skeletal muscle strength, mass and quality in the elderly. ${ }^{19}$ In contrast to Enright and Poh who failed to show a significant gender difference in 6MWD in Singaporean and Caucasian populations, ${ }^{8,10}$ the authors found a statistically significant difference in 6MWD between males and females. This inconsistency is most likely due to the dissimilarity in the age ranges and presence or absence of comorbid conditions among the study populations. Additionally, the influence of gender on the walked distance could be explained in physiological terms by the differences in heart rate response, systolic BP changes, plasma lactate levels, respiratory exchange ratio and shifts in plasma volume during exercise. $^{20,21}$ The post-walk peak heart rate in our study was relatively lower compared to previous reports. Although, in broader terms, several interlinked factors such as psycho-social, physiological and genetic variations may explain these differences and the ultimate levels of exercise, a major contributor is likely to be the low priority given to regular exercise in Bangladeshi society. This sedentary life style is likely to have the impact on peak heart rate and 6MWT of the studied subjects by affecting their perceptions of physical fitness, self efficacy (judgment of own capacity), motivation and the familiarity with and performance during physically demanding situations.

Some of the strengths of this study are that randomly selected healthy individuals were enrolled who underwent medical screening by trained physicians and assistant and included a wide age range from a geographically-related population group not studied previously. Additionally, these subjects were chosen from an ethnically diverse community of across Bangladesh, representing a range of socioeconomic and occupational status. These findings should, however, be interpreted with the certain cautions, as the subjects were volunteering adults, which may have ascertained a group who was well motivated and healthier, thus resulting in a recruitment bias. Moreover, carbon monoxide levels in these volunteers were not determined, as low level exposure has shown to curtail exercise ability in healthy individuals ${ }^{22}$. Furthermore, information on physical activity level of the individuals was not collected in this study which has shown to effect exercise capacity.

Conclusion: 6MWT is a simple, inexpensive test that can be safely performed in the outpatient setting. The mean 6MWD in the studied cohort was considerably shorter than previously published references. Gender, age and height were the most significant independent physiological determinants of 6MWD. Future studies with larger numbers incorporating models to unravel the 6MWD relationship with habits, mood, genetic factors in addition to cognitive and psychological perspectives are warranted.

\section{Limitation of study}

i) This study was conducted upon only 190 subjects

ii) Similarly on large scale and age stratified studies are needed to get the actual 6MWT and $6 \mathrm{MWD}$ value of Bangladeshi population.

\section{References}

1. Knox AJ, Morrison JF, Muers MF. Reproducibility of walking tests results in chronic obstructive airways disease. Thorax 1988; 43: 388-92.

2. ATS Committee on Proficiency Standards for Clinical Pulmonary Function Laboratories. ATS statement: guidelines for the six-minute walk test. Am J Respir Crit Care Med 2002; 166: 111-7. 
3. Woo MA, Moser DK, Stevenson LW et al. Six minute walk test and heart rate variability: lack of association in advanced stages of heart failure. Am J Respir Crit Care Med 1997; 6: 348-54.

4. Cahalin L, Pappagianopoulos P, Prevost S et al. The relationship of the 6-minute walk test to maximal oxygen consumption in transplant candidates with endstage lung disease. Chest 1995; 108: 452-9.

5. Roul G, Germain P, Bareiss P. Does the 6-minute walk test predict the prognosis in patients with NYHA class II or III chronic heart failure? Am Heart J 1998; 36: 449-57.

6. Guyatt GH, Sullivan MJ, Thompson PJ et al. The sixminute walk: a new measure of exercise capacity in patients with chronic heart failure. Can Med Assoc J 1985; 132:919-23.

7. Steffen TL, Hacker TA, Mollinger L. Age and genderrelated test performance in community dwelling elderly people: six minute walk test, Berg balance scale, timedup and go test, and gait speeds. Phys Ther 2002; 82: 128-37.

8. Enright PL, McBurnie MA, Bittner V et al. The 6minute walk test. A quick measure of functional status in elderly adults. Chest 2003; 123:387-98.

9. The World Medical Association: World Medical Association Declaration of Helsinki: ethical principles of medical research involving human subjects [Internet]. 2013. Available from: http://www.wma.net/e/policy/b3.htm

10. Poh H, Eastwood PR, Cecins NM et al. Six minute walk distance in healthy Singaporean adults cannot be predicted using reference equations derived from Caucasian populations. Respirology 2006; 11: 211-6.

11. Takishima T. Exercise Testing: Annual Report of Japanese Respiratory Failure Research Working Group. Tokyo: Ministry of Health and Welfare of Japan; 1986.
12. Teramoto S, Ohga E, Ishii $\mathrm{T}$ et al. Reference value of six-minute walking distance in healthy middle aged and older subjects. Eur Respir J 2000; 15: 1132-3.

13. Troosters T, Gosselink R, Decramer M. Six minute walking distance in healthy elderly subjects. Eur Respir J 1999; 14: 270-4.

14. Enright PL, Sherrill DL. Reference equations for the six-minute walk in healthy adults. Am J Respir Crit Care Med 1998; 158: 1384-7.

15. Rikli RE, Jones CJ. Functional fitness normative scores for community-residing older adults, ages 60-94. $J$ Aging Phys Act 1999; 7: 162-81.

16. Stevens D, Elpern E, Sharma K et al. Comparison of hallway and treadmill six-minute walk tests. $A m J$ Respir Crit Care Med 1999; 160: 1540-3.

17. Gibbons WJ, Fruchter N, Sloan S et al. Reference values for a multiple repetition 6-minute walk test in healthy adults older than 20 years. $J$ Cardiopulm Rehabil 2001; 21: 87-93.

18. Wasserman K, Hansen JE, Sue DY, editors. Principles of exercise testing and interpretation. 3rd ed. Baltimore: Williams \& Wilkins; 1999.

19. Goodpaster BH, Park SW, Harris TB et al. The loss of skeletal muscle strength, mass, and quality in older adults: the health, aging and body composition study. $J$ Gerontol A Biol Sci Med Sci 2006; 61: 1059-64.

20. Deschenes MR, Hillard MN, Wilson JA et al. Effects of gender on physiological responses during submaximal exercise and recovery. Med Sci Sports Exerc 2006; 38: 1304-10.

21. Kang J, Hoffman JR, Chaloupka EC et al. Gender differences in the progression of metabolic responses during incremental exercise. J Sports Med Phys Fitness 2006; 46: 71-8.

22. Hsia CC. Respiratory function of haemoglobin. $N$ Engl J Med 1998; 338: 239-47. 